\title{
Transcanal Endoscopic Ear Surgery for The Management of Pediatric Perilymphatic Fistula Caused by Trauma
}

\author{
Sang-Hoon Lee ${ }^{1}$, Da Hee Park², Il Woo Lee ${ }^{2}$, and Hyun Min Lee ${ }^{2}$ (i) \\ ${ }^{\prime}$ Department of Otorhinolaryngology-Head and Neck Surgery, Busan Saint Mary's Hospital, Busan; and \\ ${ }^{2}$ Department of Otorhinolaryngology-Head and Neck Surgery, Pusan National University College of Medicine, \\ Pusan National University Yangsan Hospital, Yangsan, Korea
}

\author{
내시경 귀수술을 통하여 치료한 소아 외상성 외림프 누공 1예 \\ 이상훈 ${ }^{1} \cdot$ 박다희 $^{2} \cdot$ 이일우 ${ }^{2} \cdot$ 이현민 ${ }^{2}$ \\ ${ }^{1}$ 부산성모병원 이비인후과, ${ }^{2}$ 부산대학교 의과대학 양산부산대학교병원 이비인후과학교실
}

\author{
Received June 8, 2021 \\ Revised September 17, 2021 \\ Accepted October 5, 2021 \\ Address for correspondence \\ Hyun Min Lee, MD \\ Department of Otorhinolaryngology- \\ Head and Neck Surgery, \\ Pusan National University \\ College of Medicine, \\ Pusan National University \\ Yangsan Hospital, \\ Geumo-ro 20, Mulgeum-eup, \\ Yangsan 50612, Korea \\ Tel $+82-55-360-2132$ \\ Fax $+82-55-360-2162$ \\ E-mail enthmlee@gmail.com
}

Perilymphatic fistula (PLF) is caused by leakage of perilymph through an abnormal communication between the inner and middle ear. Conservative treatment is considered in the initial stages; however, exploratory tympanotomy is performed if hearing does not improve or if dizziness persists. Transcanal endoscopic ear surgery (TEES) is considered an appropriate treatment option and is gaining popularity. We report a rare case of traumatic PLF in a 7-year-old male patient, in whom pneumolabyrinth without temporal bone fracture was diagnosed and treated by exploratory tympanotomy using TEES, and review the related research to discuss the usefulness of management using TEES for PLF.

Korean J Otorhinolaryngol-Head Neck Surg 2022;65(3):174-9

Keywords Child; Fistula; Perilymph; Transcanal endoscopic ear surgery; Trauma.

\section{Introduction}

Perilymphatic fistula (PLF) refers to an abnormal communication between the inner ear and the middle ear, which can cause acute sensorineural hearing loss, tinnitus, aural fullness, and/or vertigo. Most PLFs are caused by trauma such as temporal bone fracture, penetrating trauma, barotrauma, or iatrogenic trauma. However, the cause is often idiopathic, and the diagnosis is difficult in such cases. PLFs are generally treated conservatively. However, if symptoms persist or worsen despite conservative treatment, exploratory tympanotomy

This is an Open Access article distributed under the terms of the Creative Commons Attribution Non-Commercial License (https://creativecommons.org/licenses/by-nc/4.0) which permits unrestricted non-commercial use, distribution, and reproduction in any medium, provided the original work is properly cited. may be required for accurate diagnosis and further treatment. In most cases of traumatic PLF caused by head trauma, pneumolabyrinth is accompanied by temporal bone fracture, and hence, pneumolabyrinth without temporal bone fracture is rare. ${ }^{1,2)}$ As such, the diagnosis guidelines and treatment protocols are not clearly established for this condition due to the limited number of reported cases. ${ }^{3,4}$ Transcanal endoscopic ear surgery (TEES) was first introduced in 1993 by Thomassin, et al. ${ }^{5}$ Currently, TEES is accepted as an effective surgical method to examine the middle ear cavity for the diagnosis and treatment of several otologic diseases, including PLF.-8) TEES is less invasive, cosmetically superior, and can provide better surgical field visualization than conventional microscopic otologic surgery. ${ }^{7)}$ Here, we report a case of traumatic 
PLF in a pediatric patient with pneumolabyrinth without temporal bone fracture diagnosed and treated by exploratory tympanotomy using TEES and review the related literature.

\section{Case}

A 7-year-old male patient visited the emergency room after colliding with another person and suffering an injury in the left temporal bone area. The patient complained of severe dizziness, acute-onset hearing loss in the left ear, and aural fullness; however, no other symptoms such as otorrhea, otalgia, or ear bleeding were reported. Otologic endoscopy revealed collection of a small amount of clear fluid in the left middle ear, but no other abnormalities such as bleeding or fracture associated with the external auditory canal were observed (Fig. 1). Pure-tone audiometry (PTA) revealed mixed hearing
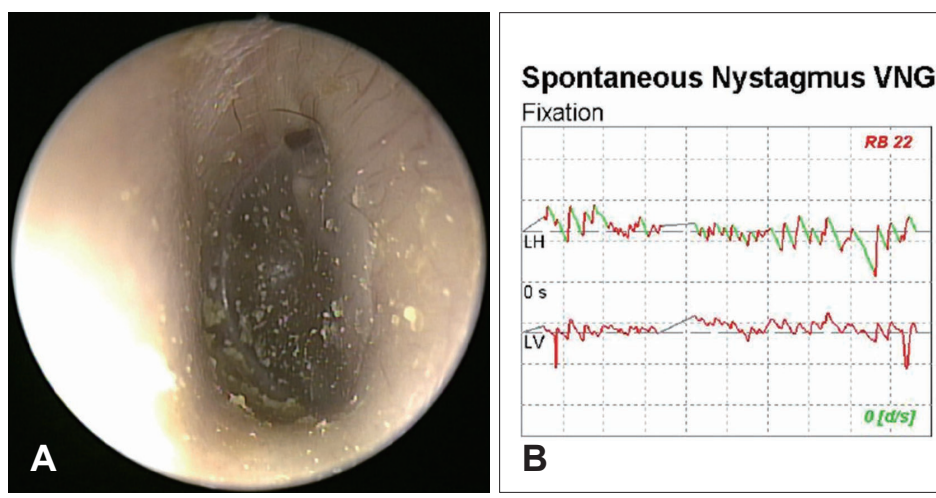

B
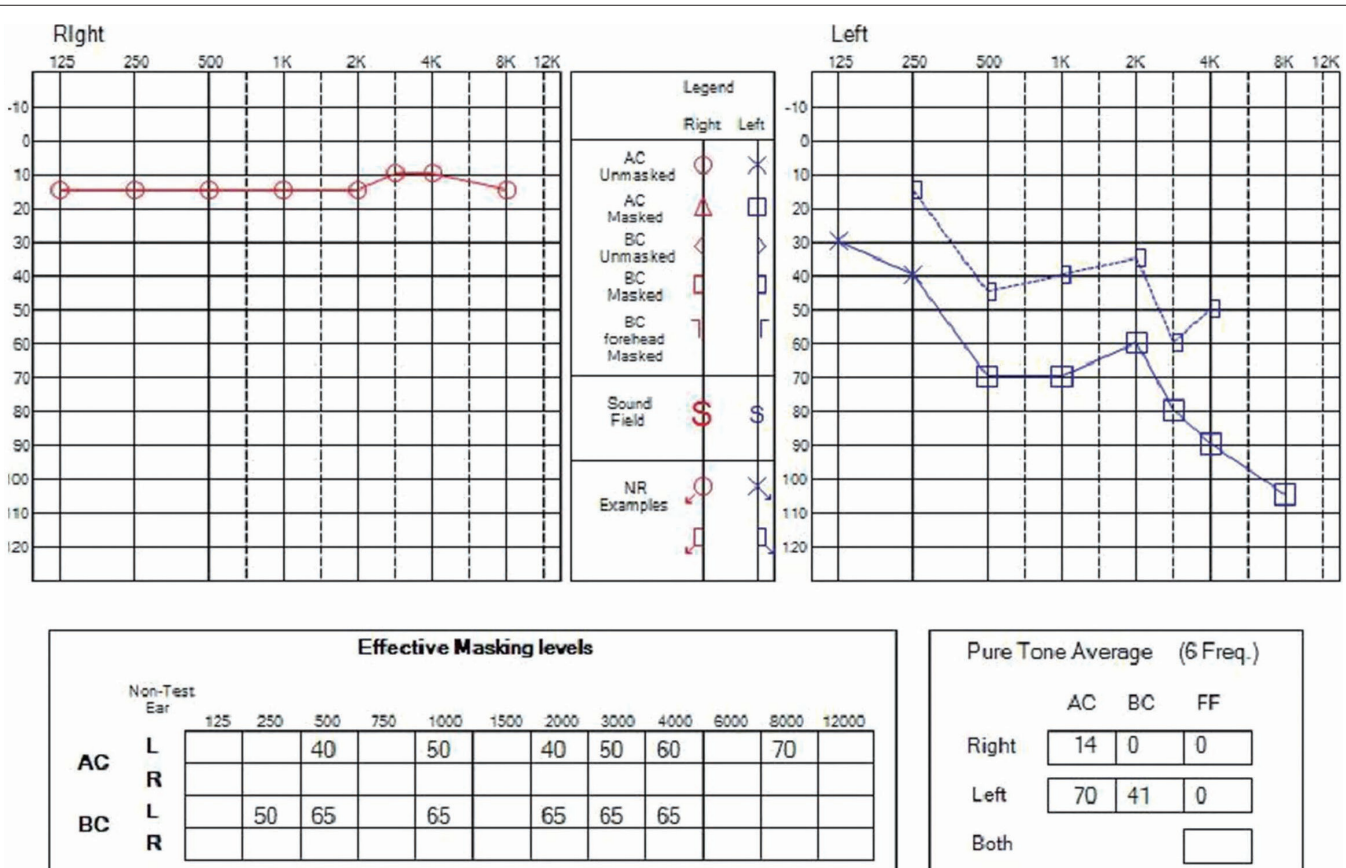

Speech Audiometer

\begin{tabular}{|l|l|l|l|l|l|}
\hline & SRT & mask & MCL & UCL & DR \\
\hline$R$ & 15 & & & & \\
\hline L & & & & & \\
\hline Binaural & & & & & \\
\hline SF & & & & & \\
\hline SF-A & & & & & \\
\hline SF-A2 & & & & & \\
\hline
\end{tabular}

\begin{tabular}{|l|c|c|c|}
\hline & $\%$ & Stimulus & mask \\
\hline R & 100 & 40 & \\
\hline L & & & \\
\hline Binaural & & & \\
\hline SF & & & \\
\hline SF-A & & & \\
\hline SF-A2 & & & \\
\hline
\end{tabular}

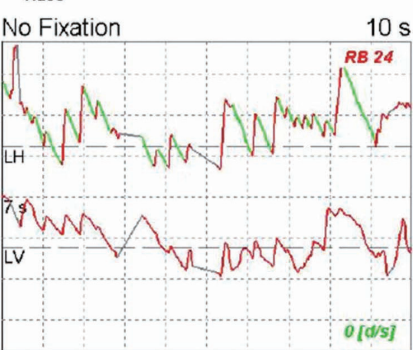

$[\mathrm{d} / \mathrm{s}]$

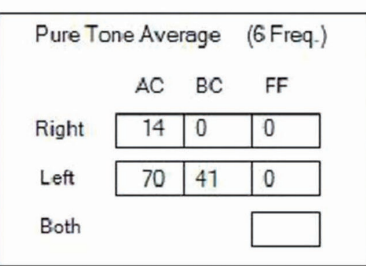

ord recognition

\begin{tabular}{|l|l|l|l|}
\hline & $\%$ & Stimulus & noise \\
\hline$R$ & & & \\
\hline$L$ & & & \\
\hline Binaural & & & \\
\hline SF & & & \\
\hline SF-A & & & \\
\hline SF-A2 & & & \\
\hline
\end{tabular}

Fig. 1. Initial Local finding, audiometry and video nystagmography of the patient. A: Initial findings of slightly clear fluid collection in the left middle ear. B: Video nystagmography showing right beating spontaneous nystagmus. C: Pure-tone audiometry showing mixed hearing loss on the left ear. 

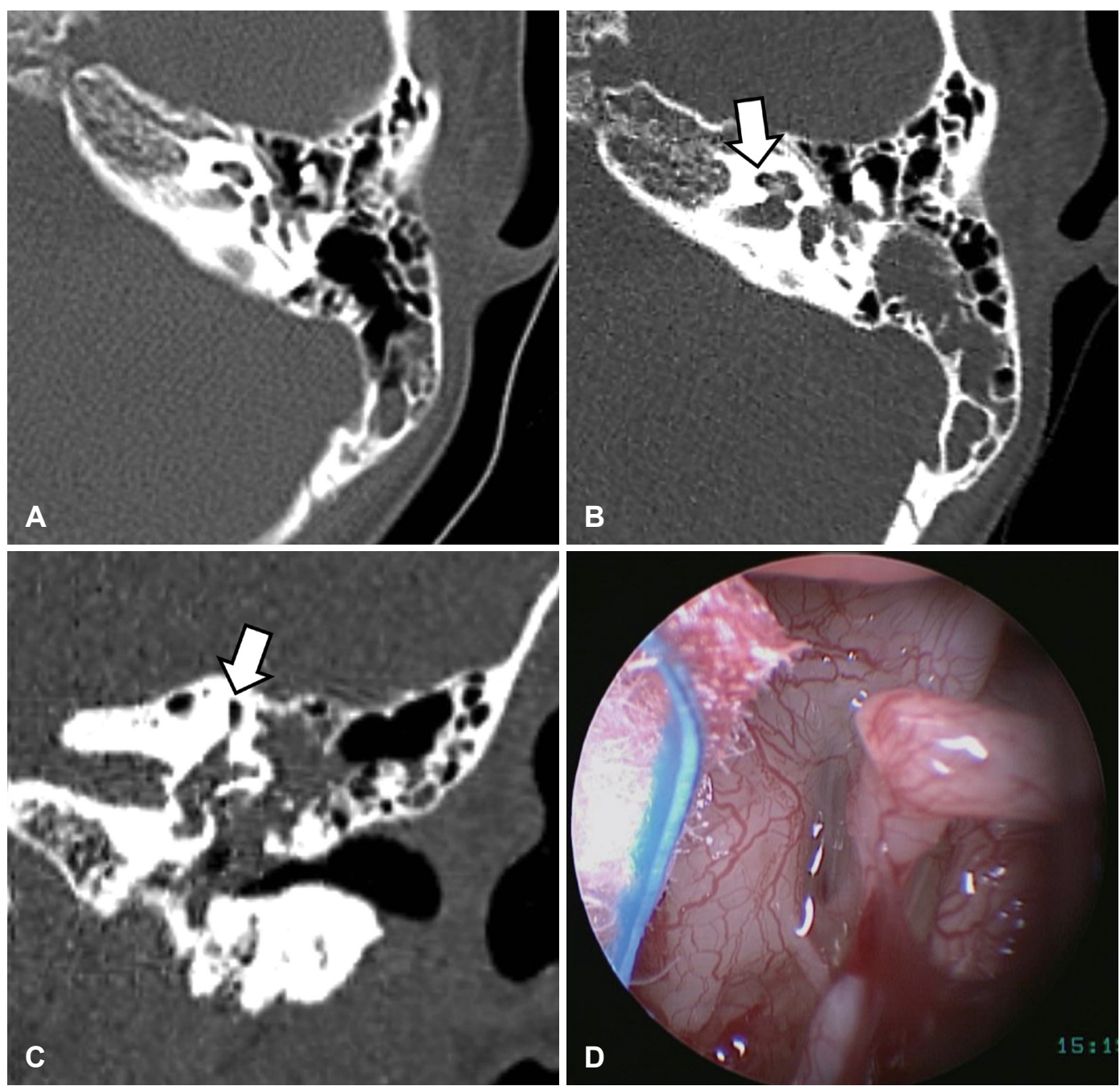

Fig. 2. Temporal bone HRCT images taken 7 hour interval after trauma and intraoperative finding of middle ear. A: Initial transverse view of HRCT showed relatively clear findings in middle ear cavity and antrum. B and C: Transverse view (B) and coronal view (C) of the HRCT obtained 7 hour after the first HRCT showing pneumolabyrinth (vestibule and cochlea) without temporal bone fracture, and increased haziness in the middle ear cavity, antrum, and mastoid air cells. White arrows indicate the pneumolabyrinth. D: Intraoperative endoscopic finding of perilymphatic fistula. Perilymph leakage can be seen in the oval window. HRCT, high-resolution computed tomography.

loss in the left ear with $70 \mathrm{~dB}$ of air-conduction (AC) hearing and $45 \mathrm{~dB}$ of bone-conduction (BC) hearing. Video nystagmography showed right beating spontaneous nystagmus, and the fistula test revealed only spontaneous nystagmus. However, the dizziness worsened when pressure was applied to the left ear (Fig. 1). Temporal bone high resolution computed tomography (HRCT) taken at another hospital immediately after the traumatic event showed relatively clear findings in middle ear cavity and antrum (Fig. 2). However, the physical examination performed at our hospital strongly suspected PLF caused by trauma, and HRCT was re-examined 7 hours after injury. Temporal bone HRCT showed the pneumolabyrinth sign in the cochlea, vestibule, and superior semicircular canal and soft tissue density in the middle ear, antrum, and mastoid air cells (Fig. 2). The patient was initially diagnosed with traumatic PLF. Conservative treatment including absolute bed rest, prophylactic antibiotics and high-dose oral steroid was initiated. The patient's complaints of hearing loss, dizziness, and spontaneous nystagmus persisted even after 3 days of conservative treatment. We performed exploratory tympanotomy using only TEES under general anesthesia 4 days after injury for the definitive diagnosis and surgical treatment of traumatic PLF. We were able to observe the middle ear cavity after elevating the tympanomeatal flap. The middle ear cavity was filled with a clear fluid, and no fracture or ossicular chain disruption was observed. The retrotympanum, which is a gravity-dependent site, exhibited refilling with the clear fluid. The middle ear cavity was carefully assessed using an en- 

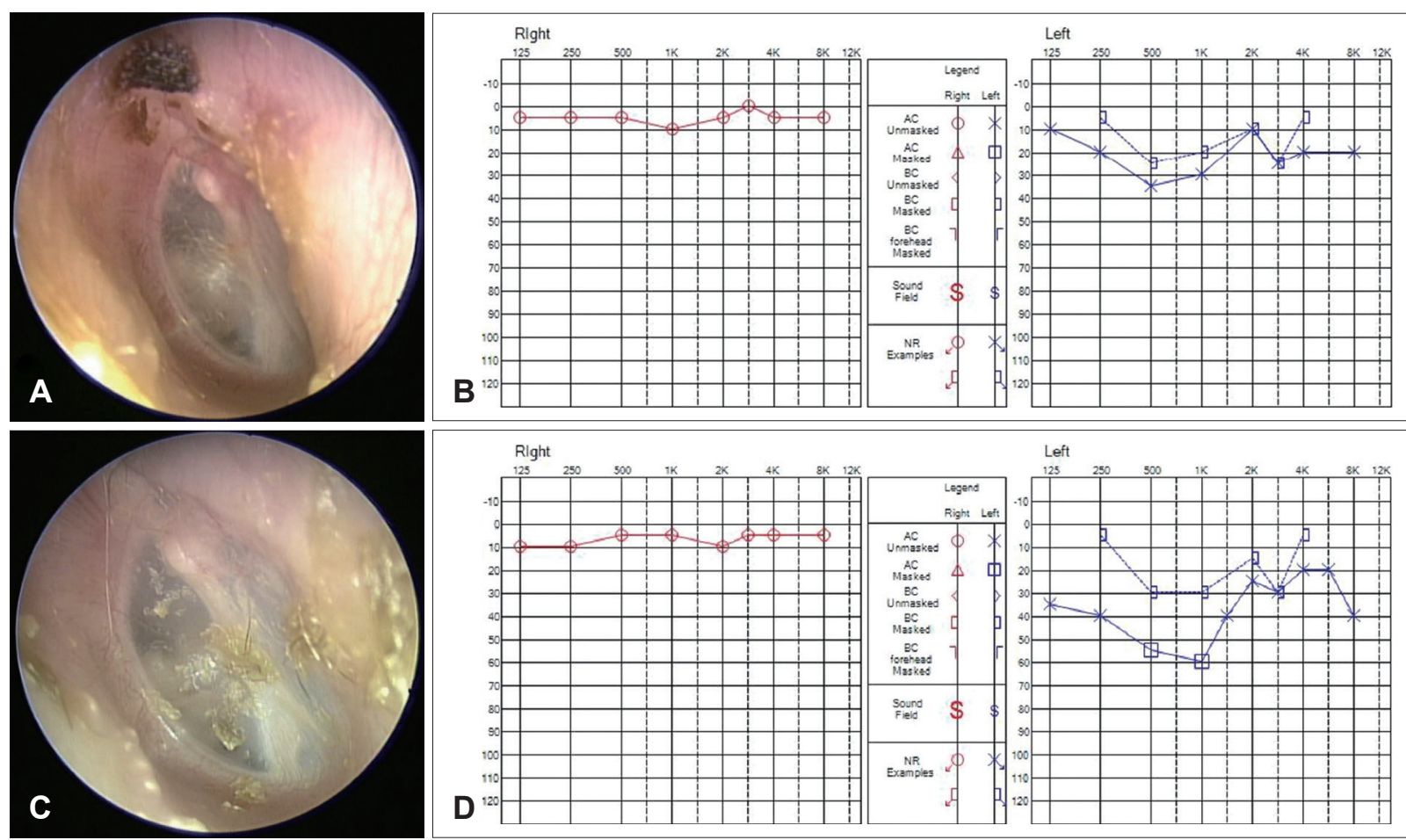

Fig. 3. Postoperative local findings and audiometic results of the patient. $A$ and $B$ : Postoperative local finding (A) and results of PTA (B) 2 months after the surgery showing relatively normal local finding and better hearing threshold. $C$ and D: Postoperative local finding (C) and PTA (D) 6 months after the surgery showing the increase in air-bone gap. PTA, pure-tone audiometry.

doscope. Perilymph leakage was not observed from the round window, but from the area between the stapes footplate and the oval window membrane (Fig. 2). The thumbnail-sized temporalis muscle fascia was harvested to seal the area around the stapes footplate to repair the fistula, and the surgery was completed after reinforcing the surrounding structures with fibrin glue and gelfoam. The dizziness improved immediately after the surgery, and spontaneous nystagmus disappeared one week after the surgery. Conservative treatment was continued, and the patient was discharged 3 weeks after the surgery. Two months after the surgery, the patient's hearing level had improved and the patient did not complain of dizziness (Fig. 3). PTA performed 6 months after the surgery revealed a hearing threshold of $41 \mathrm{~dB}$ of $\mathrm{AC}$ and $21 \mathrm{~dB}$ of $\mathrm{BC}$, and the patient did not complain of dizziness (Fig. 3). The patient is currently under outpatient follow-up without any recurrence of symptoms, one year after the surgery.

\section{Discussion}

PLF is caused by abnormal communication of the perilymph between the inner and middle ear cavity. Previously, PLF was known to occur as a postoperative complication of stapes surgery, but since Fee ${ }^{9)}$ reported traumatic PLF occurring after head trauma in 1968, trauma has been established as the most common cause of PLF. As mentioned earlier, pneumolabyrinth occurring without temporal bone fracture is rare. No definitive tests have been established for the diagnosis of PLF; therefore, the diagnosis and treatment of PLF are controversial and challenging. If a PLF is suspected, recording of a detailed medical history and performing neurotologic examination are essential. PTA in such patients may reveal conductive, sensorineural, and mixed hearing loss. PTA may show an air-bone gap (ABG), which occurs due to a third window formed by the fistula acting as a shunt for sound energy transmission to the inner ear. Spontaneous nystagmus is not common on a vestibular function test in a patient with PLF. Although nystagmus with insufflation (positive fistula test) or dizziness induced by sound (Tulio's phenomenon) may appear, the diagnostic value of these tests is not high. ${ }^{4)}$ Radiologic examinations can provide significant information regarding the condition of the inner and middle ear. HRCT is better for visualizing pneumolabyrinth than conventional CT, as it can closely evaluate the stapediovestibular joint, temporal bone fracture, and air trapping in the vestibulocochlear system. ${ }^{1)}$ However, the pneumolabyrinth could have been caused by a micro-fracture of the stapedial footplate or membrane rupture of the oval window. As in this case, even if there is no temporal bone fracture 
or hemotympanum in the initial HRCT examined after trauma, if pneumolabyrinth was seen on HRCT image or clinical symptoms are strongly suspected of PLF, delayed re-examination of HRCT may be helpful in the accurate diagnosis of PLF.

Various treatment options have been proposed for the management of PLF. Treatment is either conservative or surgical; however, the criteria for selection of a particular treatment option are controversial. For pneumolabyrinth diagnosed radiologically, as in this case, the need for exploratory tympanotomy has not been established, as pneumolabyrinth does not indicate active leakage but rather a state wherein air persists even after spontaneous healing of the fistula. ${ }^{10)}$ Improvement in vestibular symptoms and hearing have been reported through conservative treatment in patients with radiographically confirmed pneumolabyrinth. ${ }^{9,10)}$ Therefore, patients exhibiting improvement in vestibular symptoms and hearing should be treated conservatively and monitored closely. However, if vestibular symptoms persist or worsen or if hearing deteriorates despite conservative treatment, exploratory tympanotomy should be performed for definite diagnosis and surgical treatment. ${ }^{11)}$ If the site of PLF has been confirmed, it is repaired using materials such as fat, perichondrium, muscle fascia, and gelfoam. Most vestibular symptoms improve postoperatively, but hearing improves to varying degrees in such cases of PLF with pneumolabyrinth due to the permanent damage that depends on the type and severity of cochlear trauma. ${ }^{1-4)}$ Furthermore, if the pneumolabyrinth involves the cochlea, as in this case, the possibility of no improvement or even worsening of hearing despite conservative treatment is higher than if the pneumolabyrinth involves only the vestibule. ${ }^{1)}$ However, improvement in hearing and vestibular symptoms was obtained through surgical treatment in this case. With recent developments in TEES, TEES is used either alone or in combination with conventional otologic surgery using a surgical microscope for the treatment of various otologic diseases. Saxby, et al. ${ }^{8)}$ reported that an endoscope is more useful in the diagnosis and treatment of traumatic PLF due to superior surgical field visualization than a microscope. In addition, Omichi, et al. ${ }^{7)}$ reported that TEES has several advantages, including lesser surgical time than conventional surgical methods using a microscope. In the present case, the exact location of the fistula was accurately identified with TEES, which could have improved the patient's symptoms. In addition, advantages such as a reduced recovery period after surgery and reduced postoperative pain were also observed. However, a limitation of this approach is that, considering the patient's young age, con- siderable effort is required to insert the sealing material in the correct position while performing TEES with one hand to prevent any damage to the ossicular chain. In addition, the ABG had increased slightly in PTA performed 6 months after the surgery. Therefore, the possibility that postoperative adhesions occurred in the middle ear cavity cannot be excluded. In this case of traumatic PLF caused by a strong external force to the head, pneumolabyrinth was not accompanied by temporal bone fracture on HRCT. The PLF site was confirmed using TEES and repaired surgically. The patient's vestibular symptoms and hearing improved after surgical management. In cases of suspected PLF unresponsive to conservative treatment, exploratory tympanotomy should be considered. In the present case, a definite benefit in the diagnosis and management of PLF using TEES was confirmed. In the future, additional studies on the treatment of PLF using TEES are necessary.

\section{Acknowledgments}

None

\section{Author Contribution}

Conceptualization: Hyun Min Lee. Data curation: Sang-Hoon Lee, Da Hee Park. Supervision: Il Woo Lee, Visualization: Hyun Min Lee. Writing — original draft: Sang-Hoon Lee. Writing-review \& editing: Hyun Min Lee.

\section{ORCIDs}

Hyun Min Lee https://orcid.org/0000-0001-6306-5256

Il Woo Lee https://orcid.org/0000-0001-9561-6585

\section{REFERENCES}

1) Woo HJ, Song SY, Kim YD, Bai CH. Pneumolabyrinth without temporal bone fracture: Different outcomes for hearing recovery. Laryngoscope 2008;118(8):1464-6.

2) Sousa Menezes A, Ribeiro D, Miranda DA, Martins Pereira S. Perilymphatic fistula and pneumolabyrinth without temporal bone fracture: A rare entity. BMJ Case Rep 2019;12(3):e228457.

3) Lao WW, Niparko JK. Assessment of changes in cochlear function with pneumolabyrinth after middle ear trauma. Otol Neurotol 2007;28(8):1013-7.

4) Kita AE, Kim I, Ishiyama G, Ishiyama A. Perilymphatic fistula after penetrating ear trauma. Clin Pract Cases Emerg Med 2019; 3(2):115-8.

5) Thomassin JM, Korchia D, Doris JM. Endoscopic-guided otosurgery in the prevention of residual cholesteatomas. Laryngoscope 1993; 103(8):939-43.

6) Jang $\mathrm{CH}$, Choi CI. Transtympanic endoscopic diagnosis of perilymph fistula. Korean J Otorhinolaryngol-Head Neck Surg 2000;43(4):430-3.

7) Omichi R, Kariya S, Maeda Y, Nishizaki K. Transcanal endoscopic ear surgery for perilymphatic fistula after electric acoustic stimulation. Auris Nasus Larynx 2018;45(3):657-60

8) Saxby AJ, Fandino M, Kozak FK. Management of traumatic perilymph fistula with endoscopic techniques. ANZ J Surg 2018; 88(1-2):E93-4.

9) Fee GA. Traumatic perilymphatic fistulas. Arch Otolaryngol 1968;88(5):477-80. 
Perilympharic Fistula and Endoscopic Ear Surgery I Lee SH, et al.

10) Bogaerts M, Waterval J, van Dinther J, Somers T, Zarowski A, Offeciers FE. Treatment of traumatic stapediovestibular luxation: Case report with the introduction of a new technique and review of literature. Otol Neurotol 2014;35(4):582-8.
11) Prisman E, Ramsden JD, Blaser S, Papsin B. Traumatic perilymphatic fistula with pneumolabyrinth: Diagnosis and management. Laryngoscope 2011;121(4):856-9. 